\title{
Recent Advances in Understanding Delayed Photoluminescence in Colloidal Semiconductor Nanocrystals
}

\author{
Arianna Marchioro*
}

\begin{abstract}
Colloidal semiconductor nanocrystals display remarkably bright, strongly size-dependent photoluminescence properties. Following photoexcitation of these materials, temporary charge carrier separation can occur where one or both charge carriers are trapped. Charge detrapping can reform the emissive state on long time scales up to seconds, causing delayed luminescence. This delayed luminescence has not yet been thoroughly explored, and appears to be closely associated with a phenomenon observed at the single particle level, i.e. photoluminescence intermittency (blinking). Here, some of our recent work on the delayed luminescence properties of nanocrystals of different chemical composition is reviewed. These results provide insight into the mechanism of carrier detrapping, and are discussed in the context of photoluminescence blinking.
\end{abstract}

Keywords: Charge separation · Colloidal semiconductor nanocrystals · Delayed luminescence · Detrapping

\section{Introduction}

Semiconductor nanocrystals (NCs) are promising materials for a wide range of technological applications, spanning solar energy conversion, photocatalysis, bioimaging, nanophotonics, nanoelectronics and spintronics. ${ }^{[1]}$ Typically composed of an inorganic semiconducting lattice capped with organic ligands, and only several nanometers in diameter, these materials often experience quantum confinement effects and exhibit very specific properties that differ from the analogous bulk inorganic semiconductor material. In particular, these materials often show strong photoluminescence (PL), where the energy can be changed by altering the size and/or the shape of these nanocrystals, thus providing a high degree of tunability. Another important characteristic of colloidal semiconductor nanocrystals is their high surface area to volume ratio. Because of the large number of atoms that are located at the surface, the optical properties of colloidal semiconductor nanocrystals are extremely sensitive to surface chemistry. Following photoexcitation, processes such as charge trapping at the surface can potentially negatively affect the quantum yield of these materials, offering additional path-

\footnotetext{
${ }^{*}$ Correspondence: Dr. A. Marchioro

Department of Chemistry

University of Washington

Seattle, Washington 98195-1700, United States

E-mail: marchior@uw.edu
}

ways for non-radiative recombination. The situation is further complicated by the dynamic nature of the surface, where passivating ligands and surface atoms can rearrange over time, leading to fluctuations in the microscopic structure and energy of the trap states.[2,3]

A previously overlooked phenomenon has recently been recognized to play a significant role in the photophysics of semiconductor nanocrystals. 'Delayed luminescence', or the observation of very low intensity PL on timescales much longer than the intrinsic luminescence lifetime of the material, has been observed in various types of NCs. ${ }^{[4-10]}$ In these systems, delayed luminescence has been attributed to temporary charge separation, where one or both of the excitonic charges can be trapped, and eventually recombine by emission of a photon (Fig. 1).[6,7,10] Our recent work on $\mathrm{Cu}^{+}$-doped $\mathrm{CdSe}$ NCs suggests that the trapped carrier in the metastable charge-separated state is an electron, ${ }^{[6]}$ but this mechanistic scheme presented in Fig. 1 could also be applied in the case of a trapped hole. One of the intriguing aspects of this delayed luminescence is the distributed kinetics spanning from nanoseconds up to seconds. It has been shown that delayed PL decay dynamics do not follow simple exponential kinetics, but instead display distributed kinetics $^{[4-9]}$ that can in some cases be characterized by power law distributions. ${ }^{[4,8]}$ This charge-separated state has important implications for the emission properties of the materials, as it will result in an overall decrease of the time-averaged brightness of the nanocrystals. On the other hand, it also offers the possibility of tuning the duration and the magnitude of the delayed luminescence.

Many questions remain pertaining to the mechanistic pathway giving rise to the delayed luminescence component. Here, I review some of our work aimed at understanding the nature of the delayed emissive state as well as the mechanism of this process.

\section{Delayed Luminescence Kinetics}

Fig. 2 shows PL decay traces on a double-log plot for three different samples, $\mathrm{CdSe}, \mathrm{Cu}^{+}: \mathrm{CdSe}$ and $\mathrm{CuInS}_{2}$ nanocrystals. ${ }^{[10]}$ All three materials show distributed decay kinetics extending over eight orders of magnitude in time and two clearly separable time regimes. The first component (ns to sub- $\mu$ s for $\mathrm{CdSe}$, ns to tens of $\mu$ s for $\mathrm{Cu}^{+}: \mathrm{CdSe}$ and $\mathrm{CuInS}{ }_{2}$ ) corresponds to a prompt luminescence decay, where the majority of the PL decay occurs. The prompt luminescence at $20 \mathrm{~K}$ can be fitted to a single exponential and yields a lifetime of $c a .70 \mathrm{~ns}$ for undoped CdSe, $1 \mu$ s for $\mathrm{Cu}^{+}: \mathrm{CdSe}$ and $2 \mu$ s for $\mathrm{CuInS}$. A second long-lived decay component is observed after the prompt luminescence decay. This emission is referred to as delayed luminescence and remarkably, extends up to seconds. Previous reports on delayed luminescence of CdSe nanocrystals ${ }^{[8]}$ and CdSe nanoplatelets ${ }^{[9]}$ have shown similar distributed kinetics spanning over several orders of magnitude in time as well as power law kinetics (i.e. a straight line on a double log plot) for these samples. It is interesting to note that the decay kinetics presented in Fig. 2 deviate from power 


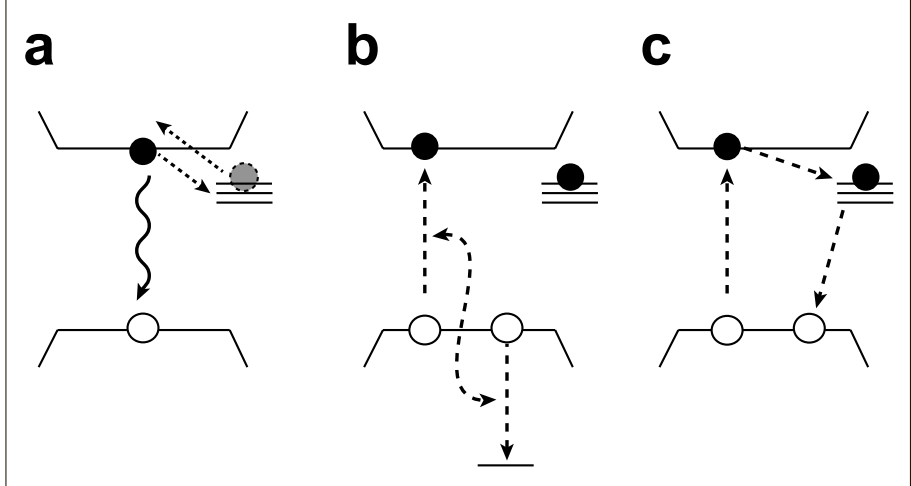

Fig. 1. Schematic illustration of the processes involved in the delayed luminescence of semiconductor nanocrystals. a) An exciton can recombine radiatively by emission of a photon. Alternatively, one of the charge carriers is trapped. For purposes of illustration, only electron trapping is depicted here, but the mechanistic scheme would be equally viable in the case of a trapped hole. Electron detrapping eventually occurs to reform the emissive state. This process takes place with very distributed kinetics. b) and c) illustrate various non-radiative recombination processes: photoexcitation of a nanocrystal already in its metastable charge-separated state can lead to nonradiative recombination, where the nanocrystals appears dark. b) shows a generic Auger non-radiative process, c) illustrates a Shockley-ReadHall recombination mechanism to return to the original state. Similar $\mathrm{a}, \mathrm{b}$ and $\mathrm{c}$ ) processes occurring at the single-nanocrystal level could explain photoluminescence blinking behavior observed in colloidal semiconductor NCs. A single NC appears dark until charge carrier detrapping occurs. Repetitive photoexcitation of the same single nanocrystals in a charge-separated state leads to non-radiative recombination.

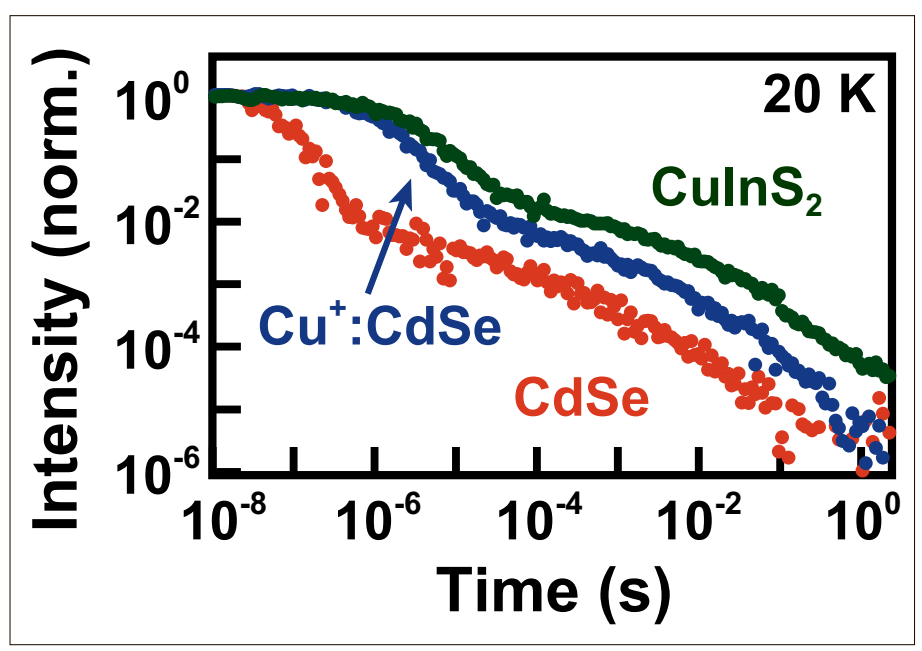

Fig. 2. Double-log plot of $\mathrm{PL}$ intensity decay for $\mathrm{CdSe}, \mathrm{Cu}^{+}: \mathrm{CdSe}$, and

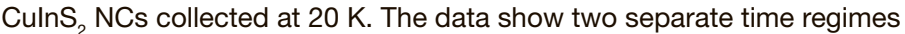
associated to prompt and delayed luminescence regimes at short and long times, respectively. Distributed decay kinetics extending up to seconds is observed for all three materials. The CdSe NCs exhibit faster prompt decay, and as consequence, less delayed luminescence than either the $\mathrm{CuInS}_{2}$ or $\mathrm{Cu}^{+}: \mathrm{CdSe}$ NCs. A longer prompt lifetime is likely to increase the trapping probability to the metastable state, overall increasing the total amount of delayed luminescence. Adapted with permission from ref. [10]. Copyright 2016 American Chemical Society. law for all samples. This deviation from power law behavior was first reported by our group and not observed previously, possibly due to the data being collected on a shorter time window. ${ }^{[10]}$

From these results, another important observation can be made: under similar measurement conditions, $\mathrm{CdSe} \mathrm{NCs}$ exhibit substantially less delayed luminescence than $\mathrm{Cu}^{+}: \mathrm{CdSe}$ or $\mathrm{CuInS}_{2}$ NCs. We proposed that this difference arises from the competition between prompt luminescence and formation of a metastable charge-separated state, and is directly related to the prompt lifetime of the material. Indeed, NCs with longer lifetime will have increased trapping probability to a metastable charge-separated state.

The data presented in Fig. 2 reveal a common feature in the photophysics of colloidal chalcogenide-based semiconductor nanocrystals. Such delayed luminescence is likely to be a characteristic occurrence among colloidal semiconductor nanocrystals, and could serve as a versatile experimental tool to investigate the nature of trap states in these materials.

\section{Delayed Luminescence and Relationship to Blinking}

Blinking, or fluorescence intermittency, refers to the observation of nonemissive periods at the single particle level under continuous excitation. This phenomenon is not specific to nanocrystals, and has also been observed in molecular[11] and biological systems. ${ }^{[12]}$ As a result, PL intermittency is characterized by dark 'off' states and bright 'on' states. ${ }^{[13]}$ The statistics of these 'on' and 'off' states in single semiconductor NCs have previously been described by distributed kinetics, most commonly by fitting to a powerlaw. ${ }^{[14,15]}$ This PL intermittency in NCs has been studied extensively and several models have been proposed to explain this phenomenon. ${ }^{[16-20]}$ Many of them explain the 'off' state in terms of charge carrier localization. ${ }^{[21,22]}$ This charge carrier localization leaves the $\mathrm{NC}$ in a charged state, thus favoring Auger or other nonradiative processes during the successive photoexcitation event (Fig. 1). The idea that the delayed luminescence phenomenon might be related to PL blinking at the single particle level was first proposed in 2008. ${ }^{[4]}$ Based on the observations of similar power law dynamics in fluorescence ensemble measurements and single particle measurements, it was hypothesized that fluorescence blinking on single semiconductor nanocrystals and fluorescence decay in an ensemble originate from the same charge trapping event. ${ }^{4]}$ Several successive reports suggested a mechanistic link between the two phenomena. ${ }^{[6,8]}$ Work performed in our group showed for the first time overlapping timescales of the blinking 'off' dynamics and of the ensemble delayed luminescence. ${ }^{[7]}$ Fig. 3 plots delayed luminescence decay at room temperature for $\mathrm{CuInS}_{2}$ nanocrystals, together with the nonemissive ('off') blinking statistics collected on many single nanocrystals from the same ensemble. Although the quantitative intensity scaling is arbitrary (due to the conditions of the two different experiments), we observe that delayed luminescence and blinking both show remarkably similar distributed kinetics, extending over many orders of magnitude in time.

Overlapping timescales for both processes were not experimentally reported previously, and this observation supports the proposal of a mechanistic link between the delayed ensemble PL and the singleparticle PL blinking. Additionally, photoexcitation power-dependence measurements showed saturation of the delayed luminescence intensities starting at relatively low powers. ${ }^{[7]}$ This observation indicates that photoexcitation of $\mathrm{NCs}$ already in the metastable state leads to efficient nonradiative decay (Fig. 1). 


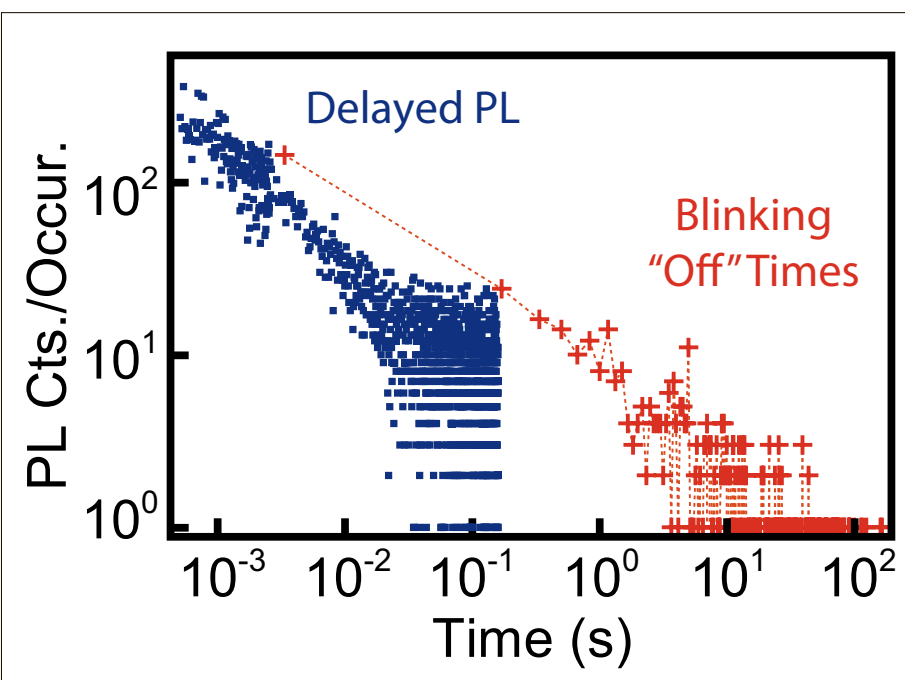

Fig. 3. Double-log plot of $\mathrm{PL}$ intensity decay for Culns $s_{2}$ CdS NCs (blue squares) and histogram of blinking 'off' statistics (red crosses). The dashed line is a guide to the eye. All data were collected at room temperature. Adapted with permission from ref. [7]. Copyright 2016 American Chemical Society.

\section{Delayed Luminescence Mechanism}

It was initially unclear if the emission originated directly from recombination of the trapped carrier, i.e a donor-acceptor pair type of mechanism, or if the emission involved release of the trapped carrier and successive reformation of the prompt emissive state. The nature of the emissive state for this delayed luminescence was first investigated in $\mathrm{Cu}^{+}: \mathrm{CdSe}^{[6]}$ The spectrum associated with the delayed luminescence was found to be similar to the one associated to the prompt luminescence, suggesting that the same luminescent excited state is reformed after the metastable chargeseparated state. However, in that case the spectral signals were partly beyond the detection range of our instruments. ${ }^{[6]}$ In a recent study, the authors compared prompt and delayed excitonic emission spectra of an ensemble of CdSe NCs and found essentially identical spectra for luminescence collected during the prompt lifetime of the material and luminescence collected and long time delays after excitation. ${ }^{[8]} \mathrm{A}$ small $(10 \mathrm{meV})$ redshift was observed and attributed to particle size inhomogeneity. Smaller NCs have shorter lifetime, thus contributing to a lesser extent to the delayed luminescence observed on long timescales.

Recent time-resolved magneto-PL (MCPL) measurements in our group support these conclusions for different samples. ${ }^{[10]}$ Figs 4A,B show magnetic circularly polarized luminescence spectra of $\mathrm{Cu}^{+}: \mathrm{CdSe}$ and $\mathrm{CuInS}_{2}$ at $20 \mathrm{~K}$ and $6 \mathrm{~T}$. We reported previously singlettriplet excited-state exchange splittings for both $\mathrm{Cu}^{+}: \mathrm{CdSe}$ and $\mathrm{CuInS}{ }_{2} \mathrm{NCs} .{ }^{[23]}$ At low temperature and presence of a magnetic field, the triplet state is split into its Zeeman components, giving rise to a partially circularly polarized emission. The difference between the left and right circularly polarized emission, normalized by the total intensity (i.e. the polarization ratio), can be monitored and both $\mathrm{Cu}^{+}: \mathrm{CdSe}$ and $\mathrm{CuInS}$, show a significant polarization ratio in their prompt emission. The same experiment has been performed
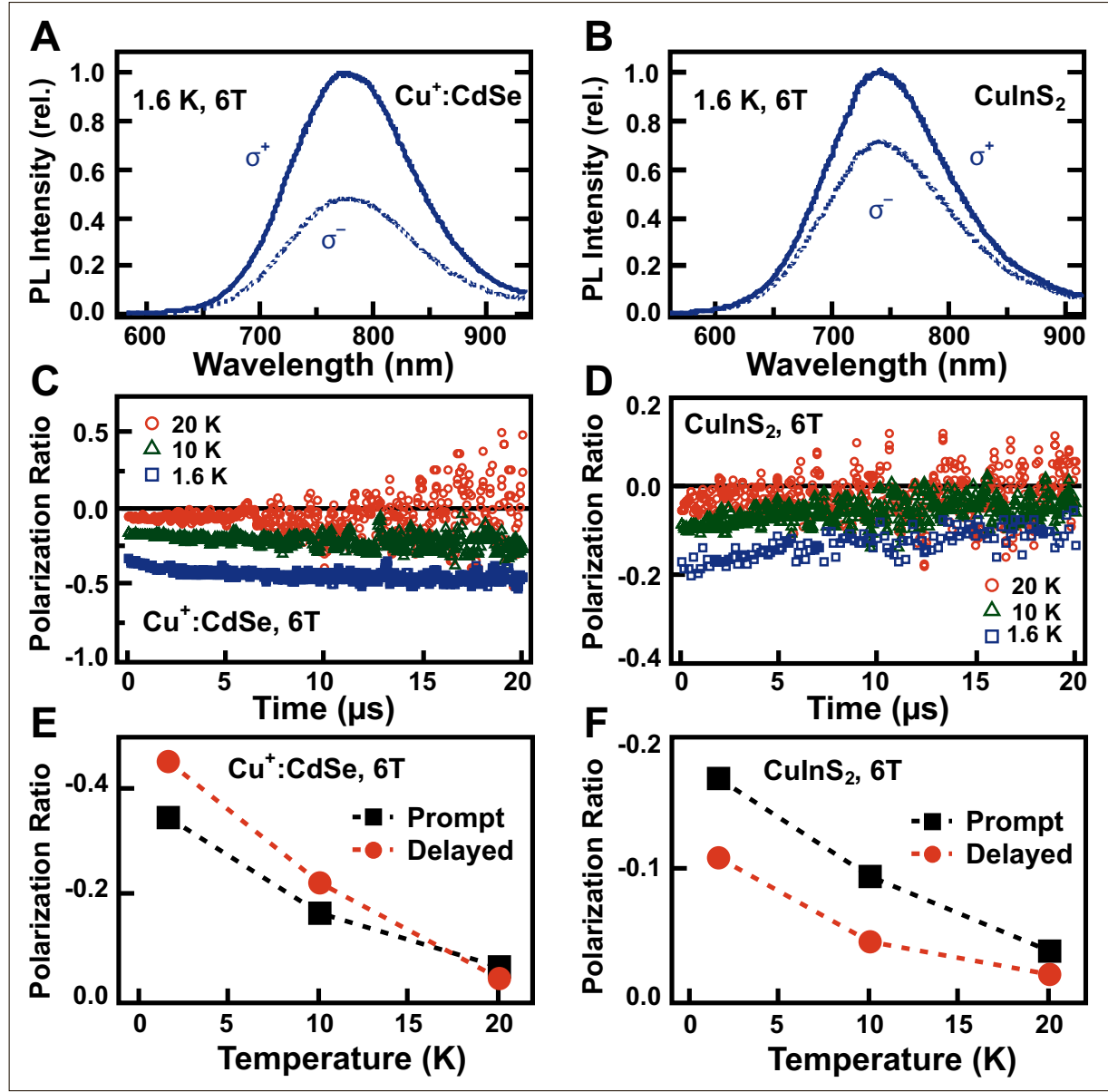

Fig. 4. Magnetic circularly polarized photoluminescence (MCPL) spectra of (A) $\mathrm{Cu}^{+}$:CdSe and (B) CulnS ${ }_{2}$ NCs measured at $1.6 \mathrm{~K}$, with an applied magnetic field of $6 \mathrm{~T}$. Solid line: right circularly polarized, $\sigma^{+}$. Dashed line: left circularly polarized, $\sigma^{-}$. (C,D) MCPL polarization ratio at $6 \mathrm{~T}(\Delta / / \mathrm{l}$, measured at the prompt luminescence maximum) plotted vs luminescence decay time measured at $1.6 \mathrm{~K}$ (blue squares), $10 \mathrm{~K}$ (green triangles), and $20 \mathrm{~K}$ (red circles) for (C) $\mathrm{Cu}^{+}$: $\mathrm{CdSe}$ and (D) CulnS $_{2}$ nanocrystals. (E,F) $\Delta / / /$ plotted vs temperature for prompt (black) and delayed (red) luminescence. The delayed luminescence values represent $\Delta / /$ averaged between 10 and $20 \mu s$ after the end of the excitation pulse, well after the decay of the prompt luminescence. Adapted with permission from ref. [10]. Copyright 2016 American Chemical Society. 
These results conclusively eliminate the possibility of photoluminescence being due to donor-acceptor pair recombination. If delayed luminescence occurs through charge detrapping, what mechanism is responsible for charge detrapping to reform the emissive state? In order to answer this question, temperature-dependent measurements were performed on CdSe, $\mathrm{Cu}^{+}: \mathrm{CdSe}$ and $\mathrm{CuInS}_{2} \cdot{ }^{[10]}$ Figs 5A,B show delayed luminescence decay kinetics for $\mathrm{Cu}^{+}: \mathrm{CdSe}$ and $\mathrm{CuInS}_{2}$. The long-time dynamics for the delayed luminescence do not appear to change with increasing temperature up to $200 \mathrm{~K}$, as observed on a double log plot (Figs 5A,B) and on linear plots.[10] Above $200 \mathrm{~K}$, the decay kinetics does start changing, indicating a new thermally activated non-radiative decay channel. Interestingly, the delayed luminescence amplitude decreases substantially with increasing temperature. We attribute this trend to the change in lifetime of the excited state. Figs 5C,D show the integrated delayed luminescence (all the photons integrated after termination of the excitation pulse and after complete decay of the prompt luminescence) as a function of temperature, and the prompt luminescence lifetime is plotted for comparison. $\mathrm{Cu}^{+}: \mathrm{CdSe}$ shows increased prompt luminescence lifetime with decreasing temperature, consistent with a stabilization of a triplet state at lower temperatures. The temperature dependence of the integrated delayed luminescence shows similar trend, indicating that longer lifetimes lead to larger amounts of delayed luminescence. This observation confirms the trend observed in Fig. 2 for different samples, where the amount of delayed luminescence is determined by the prompt luminescence lifetime, specific to each material.

Several important observations can be made on the delayed luminescence dynamics. As already illustrated in Fig. 2, the double-log plots of the delayed luminescence decay data show significant curvature, suggesting that the underlying distribution of rate constants cannot be described accurately by power-law expressions. Moreover, the dynamics of delayed luminescence are temperature independent from $20 \mathrm{~K}$ to $200 \mathrm{~K}$. This implies that the rate-limiting step for detrapping must involve electron (or hole) tunneling. Using a simple kinetic model where the delayed luminescence is described by a tunneling process with a Gaussian distribution of barrier widths, which is equivalent to use a log-normal distribution of tunneling rate constants, we showed that the data can be fitted remarkably well (solid lines in Fig. $5 \mathrm{~A}) .{ }^{[10]}$ From a physical perspective, such a distribution in barrier widths would correspond to a distribution in the displacement

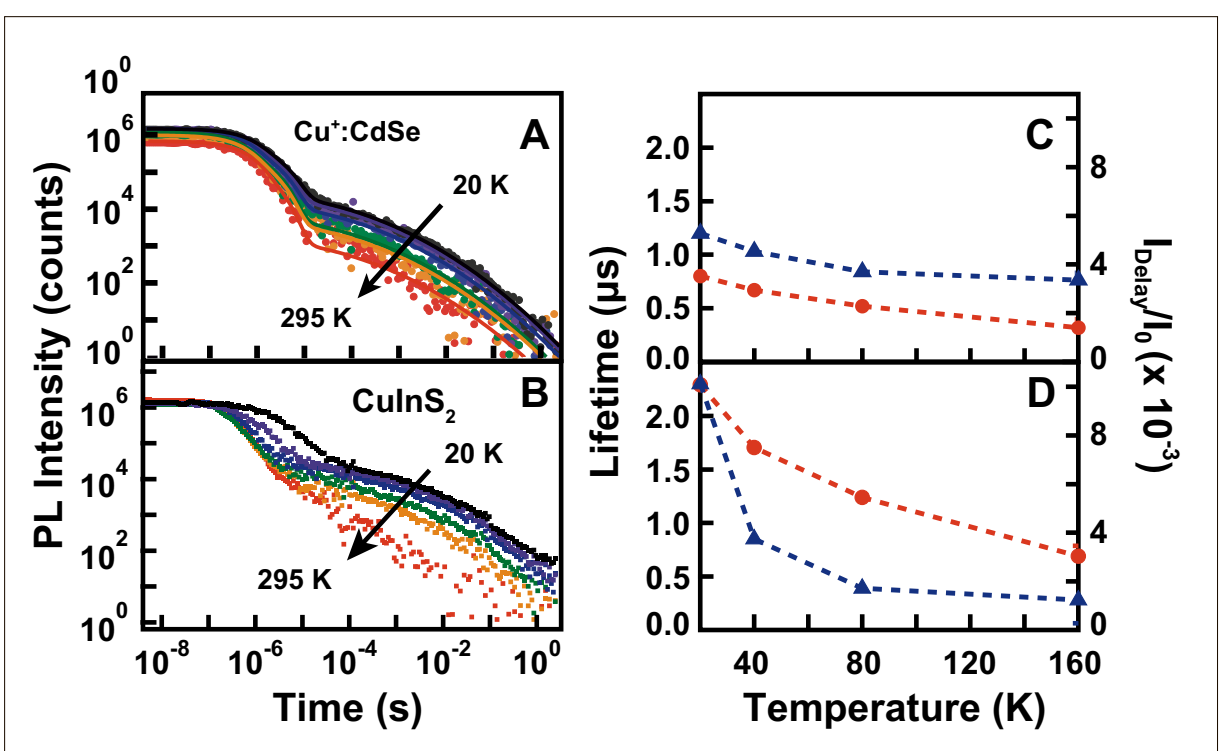

Fig. 5. (A, B) Double-log plots of PL intensity decay for (A) $\mathrm{Cu}^{+}$:CdSe NCs, and (B) CulnS $\mathrm{NCs}_{2}$ collected from 20 to $295 \mathrm{~K}$. Color code: black $=20 \mathrm{~K}$, purple $=40 \mathrm{~K}$, green $=80 \mathrm{~K}$, blue $=160 \mathrm{~K}$, yellow $=200 \mathrm{~K}$, red $=295 \mathrm{~K}$. Lines on $(\mathrm{A})$ represent simulated luminescence decay curves reproducing the $\mathrm{Cu}^{+}: \mathrm{CdSe} \mathrm{NC}$ data, obtained using a tunneling kinetic model. The first $160 \mu \mathrm{s}$ of the prompt luminescence decay at each temperature was fitted to a sum of two exponentials. The delayed luminescence was modeled as a tunneling process using a Gaussian distribution in tunneling barrier widths. (C, D) Temperature dependence of prompt luminescence decay lifetimes from single-exponential fits (blue triangles,) and the ratio of delayed to steady-state luminescence intensities $\left(I_{\text {delayed }} / I_{0}\right.$, red circles), where $I_{0}$ is the steady-state luminescence intensity measured during photoexcitation and $I_{\text {delayed }}$ is the luminescence intensity measured $450 \mu$ s after termination of the photoexcitation. (C) $\mathrm{Cu}^{+}:$CdSe NCs, and (D) CulnS $\mathrm{NCs}_{2}$. Adapted with permission from ref. [10]. Copyright 2016 American Chemical Society.

of the charge separated state's potential energy surface relative to the emissive state's surface along the tunneling coordinate.

The good agreement between this tunneling kinetic model and the temperature-independent delayed luminescence decay dynamics supports the hypothesis of a tunneling mechanism for charge detrapping. Interestingly, a tunneling mechanism has been proposed to describe blinking 'off' statistics. 'Off' statistics, which reflect detrapping dynamics analogous to the delayed luminescence decay dynamics, also appeartobe temperatureindependent. ${ }^{[14,15,24]}$ strengthening the link between the two phenomena presented above.

\section{Conclusion}

Delayed luminescence is observed across a variety of colloidal semiconductor NC materials. This long-lived emission is observed on timescales from microsecond to seconds and is attributed to temporary charge carrier trapping in a metastable state. Delayed luminescence decay traces show very distributed kinetics and deviation from power law behavior. Ensemble delayed luminescence dynamics are shown to overlap in time with blinking 'off' statistics at the single particle level, suggesting a similar microscopic origin for the two phenomena. Our data reveal that the delayed luminescence occurs through detrapping and reformation of the original emissive state, rather than through a donor-acceptor mechanism. Temperature independence of the delayed luminescence kinetics points to a tunneling mechanism for the charge carrier detrapping. A kinetic model involving a log-normal distribution of tunneling rates reproduces the data well, overall corroborating the analogy between blinking at the single particle level and delayed luminescence in ensemble measurements. Delayed luminescence is proposed as a complementary approach to blinking measurements to investigate charge trapping and detrapping dynamics in colloidal semiconductor nanocrystals.

\section{Acknowledgments}

The results presented here were made possible by the hard work of Dr. Patrick J. Whitham, Prof. Kathryn E. Knowles, and Mr. Troy B. Kilburn. I would like to thank Dr. Emily Tsui for valuable discussions. A special thanks is due to Prof. Daniel R. Gamelin for advice and support, and for having hosted me during my postdoctoral stay. Additionally, I would like to acknowledge an Early Postdoc Mobility fellowship from the Swiss National Science Foundation that financed my postdoctoral research at the University of Washington.

Received: December 3, 2016 
[1] M. V. Kovalenko, L. Manna, A. Cabot, Z. Hens D. V. Talapin, C. R. Kagan, V. I. Klimov, A L. Rogach, P. Reiss, D. J. Milliron, P. GuyotSionnnest, G. Konstantatos, W. J. Parak, T. Hyeon, B. A. Korgel, C. B. Murray, W. Heiss, ACS Nano 2015, 9, 1012.

[2] J. R. McBride, T. J. Pennycook, S. J. Pennycook, S. J. Rosenthal, ACS Nano 2013, 7, 8358

[3] O. Voznyy, S. M. Thon, A. H. Ip, E. H. Sargent, J. Phys. Chem. Lett. 2013, 4, 987.

[4] P. H. Sher, J. M. Smith, P. A. Dalgarno, R J. Warburton, X. Chen, P. J. Dobson, S. M Daniels, N. L. Pickett, P. O’Brien, Appl. Phys. Lett. 2008, 92, 101111.

[5] M. Jones, S. S. Lo, G. D. Scholes, Proc. Natl. Acad. Sci. USA 2009, 106, 3011.

[6] P. J. Whitham, K. E. Knowles, P. J. Reid, D. R. Gamelin, Nano. Lett. 2015, 15, 4045.

[7] P. J. Whitham, A. Marchioro, K. E. Knowles, T. B. Kilburn, P. J. Reid, D. R. Gamelin, J. Phys. Chem. C 2016, 120, 17136.

[8] F. T. Rabouw, M. Kamp, R. J. A. van DijkMoes, D. R. Gamelin, A. F. Koenderink, A. Meijerink, D. Vanmaekelbergh, Nano. Lett 2015, 15, 7718 .
[9] F. T. Rabouw, J. C. van der Bok, P. Spinicelli, B. Mahler, M. Nasilowski, S. Pedetti, B. Dubertret, D. Vanmaekelbergh, Nano. Lett. 2016, 16, 2047.

[10] A. Marchioro, P. J. Whitham, K. E. Knowles, T. B. Kilburn, P. J. Reid, D. R. Gamelin, J. Phys. Chem. C 2016, 120, 27040.

[11] E. A. Riley, C. M. Hess, J. R. L. Pioquinto, W. Kaminsky, B. Kahr, P. J. Reid, J. Phys. Chem. B 2013, 117, 4313 .

[12] R. M. Dickson, A. B. Cubitt, R. Y. Tsien, W. E. Moerner, Nature 1997, 388, 355.

[13] M. Nirmal, B. O. Dabbousi, M. G. Bawendi, J. J. Macklin, Nature 1996, 383, 802.

[14] M. Kuno, D. P. Fromm, H. F. Hamann, A. Gallagher, D. J. Nesbitt, J. Chem. Phys. 2000, $112,3117$.

[15] M. Kuno, D. P. Fromm, H. K. Hamann, A. Gallagher, D. J. Nesbitt, J. Chem. Phys. 2001, 115, 1028.

[16] M. Kuno, D. P. Fromm, S. T. Johnson, A. Gallagher, D. J. Nesbitt, Phys. Rev. B 2003, 67, 125304.

[17] R. Verberk, A. M. van Oijen, M. Orrit, Phys. Rev. B 2002, 66, 233202.
[18] P. A. Frantsuzov, R. A. Marcus, Phys. Rev. B 2005, 72, 155321.

[19] J. Tang, R. A. Marcus, Phys. Rev. Lett. 2005, 95, 107401 .

[20] M. Pelton, G. Smith, N. F. Scherer, R. A Marcus, Proc. Natl. Acad. Sci. USA 2007, 104 14249

[21] C. Galland, Y. Ghosh, A. Steinbrück, M Sykora, J. A. Hollingsworth, V. I. Klimov, H. Htoon, Nature 2012, 479, 203.

[22] W. Qin, P. Guyot-Sionnest, ACS Nano 2012, 6 , 9125.

[23] K. E. Knowles, H. D. Nelson, T. B. Kilburn, D. R. Gamelin, J. Am. Chem. Soc. 2015, 137 13138.

[24] K. T. Shimizu, R. G. Neuhauser, C. A. Leatherdale, S. A. Empedocles, W. K. Woo, M. G. Bawendi, Phys. Rev. B 2001, 63, 205316. 